\title{
Modeling supersonic heated jet noise at fixed jet Mach number using an asymptotic approach for the acoustic analogy Green's function and an optimized turbulence model
}

\author{
M. Z. Afsar * \\ Department of Mechanical \& Aerospace Engineering, University of Strathclyde, Glasgow, G1 I XJ \\ A. Sescu ${ }^{\dagger}$ \\ Department of Aerospace Engineering, Mississippi State University, Starkville, MS \\ E. Minisci \\ Department of Mechanical \& Aerospace Engineering, University of Strathclyde, Glasgow, G1 I XJ
}

\begin{abstract}
In this study we show how accurate jet noise predictions can be achieved within Goldstein's generalized acoustic analogy formulation for heated and un-heated supersonic jets using a previously developed asymptotic theory for the adjoint vector Green's function and a turbulence model whose independent parameters are determined using an optimization algorithm . In this approach, mean flow non-parallelism enters the lowest order dominant balance producing enhanced amplification at low frequencies, which we believe corresponds to the peak sound at small polar observation angles. The novel aspect of this paper is that we exploit both mean flow and turbulence structure from existent Large Eddy Simulations database of two axi-symmetric round jets at fixed jet Mach number and different nozzle temperature ratios to show (broadly speaking) the efficacy of the asymptotic approach. The empirical parameters that enter via local turbulence length scales within the algebraic-exponential turbulence model are determined by optimizing against near field turbulence data post-processed from the LES calculation. Our results indicate that accurate jet noise predictions are obtained with this approach up to a Strouhal number of 0.5 for both jets without introducing significant empiricism.
\end{abstract}

\section{Introduction}

EROACOUSTIC modeling of jet noise within Goldstein's generalized acoustic analogy (GAA) [1] formulation involves A (a) solving the adjoint linearized Euler equations to determine the Green's function that defines the so-called propagator tensor and (b) appropriately modeling the Reynolds stress auto-covariance using experimental data and/or numerical simulations [1]. The far-field sound is then determined by the volume integral of an inner product of the propagator and the Reynolds stress auto-covariance tensors. This approach has proven to be successful for a number of test cases involving axi-symmetric round jets at a variety of acoustic Mach numbers and observation angles, Goldstein \& Leib [2] (hereafter referred to as G\&L) and [3]). It has also shed light on what impact the mean flow field has on the far-field radiated sound for both heated and unheated flows [4].

G\&L's predictions were computed at $O(1)$ (i.e., arbitrary) frequencies for a propagator tensor based on a weakly non-parallel mean flow. Non-parallelism appeared in the analysis at supersonic speeds and only affected the solution within a thin critical layer where the adjoint vector Green's function is singular for the locally parallel mean flow. G\&L constructed a uniformly valid composite solution for the adjoint Green's function, thus eliminating the critical-layer singularity that occurs when the observation angle, $\theta$, is close to the downstream jet axis. They showed that, as $\theta \rightarrow 0$, the dominant contribution to the propagator comes from the radial derivative of the Fourier transformed adjoint Green's function for the streamwise momentum perturbation. This was also confirmed by Karabasov et al. [5]'s numerical calculations and by Afsar et al. [3] who, together with G\&L, showed that the acoustic efficiently of this term is raised to a dipole at low frequencies even though it appears as a quadrupole when multiplied by the appropriate Reynolds stress auto-covariance component in the acoustic spectrum formula [6].

\footnotetext{
*Chancellor's Fellow. AIAA Member.

'Associate Professor, and AIAA Associate Fellow.

$\doteqdot$ Senior Lecturer. AIAA Member.
} 


$\begin{array}{llllll}\text { Experimental [13] } & \text { LES case } & \text { Description } & M_{j} & T R & M_{a} \\ \text { B118 } & \text { A1 } & \text { isothermal ideally-expanded } & 1.5 & 1.0 & 1.5 \\ \text { B122 } & \text { A2 } & \text { heated ideally-expanded } & 1.5 & 1.74 & 1.98 \\ & \text { Table 1 } & \text { Brès et al (2012) [12] test cases } & & & \end{array}$

Physically, this behavior is due to the streamwise-radial, or ' $1-2$ ', component of the fluctuating Reynolds stress multiplying the commensurate propagator component in such a manner that the latter possesses a pre-factor with directionality that scales as $\cos ^{4} \theta /(1-M(r) \cos \theta)^{6}$ (where $M(r)$ is the local Mach number profile), which, obviously, peaks as $\theta \rightarrow 0$ (where $\theta$ is the polar observation angle), thus allowing an accurate prediction to be made of the peak jet noise that experiments show usually occurs at $\theta=30^{\circ}$ in the forward arc. This result was derived in the matched-asymptotic-expansion sense, in which the solution for the pressure-like Green's function is divided into an inner region where the scaled radial coordinate $r / \sigma=O(1)$ and an $r=O(1)$ outer region with the scaled frequency, $\sigma=k_{\infty}^{*} \delta^{*} \ll O(1)$, being an asymptotically small parameter everywhere in the flow ( $\delta^{*}$ is an appropriate dimensional length scale such as the nozzle radius and $k_{\infty}$ is far-field wavenumber). Parallel flow asymptotics also showed that the components of the fluctuating Reynolds stress (other than '1-2') make a more dominant contribution to the large-angle-radiated sound at $O(1)$ frequencies under a general axi-symmetric representation of the Reynolds stress auto-covariance tensor (such as that in [7]). This picture of jet noise is, however, oversimplified because it does not take into account mean flow spreading, which Karabasov et al. [8] showed can be important at $O(1)$ Mach numbers and can increase the low-frequency radiation by as much as 8 Decibels $(\mathrm{dB})$ for $\theta=30^{\circ}$ on a subsonic jet compared to the equivalent parallel flow solution of the GAA (adjoint linearized Euler) equations.

Goldstein, Sescu \& Afsar [9] (referred to herein as GSA) constructed an asymptotic solution to the adjoint vector Green's function problem starting from the GAA equations. As opposed to low-frequency asymptotics in a parallel flow discussed above, they considered a slowly diverging jet flow in which the spread rate, $\epsilon$, is an asymptotically small parameter, $\epsilon \ll O(1)$. GSA determined that the only distinguished limit that could produce leading order changes to the acoustic spectrum is when the Strouhal number is of the same order as the jet spread rate. The resulting adjoint vector Green's function (and therefore the dominant '1-2' propagator component mentioned above) was different from the parallel flow result everywhere in the jet (not just in the critical layer as in [2]). Following on from GSA, Afsar et al. [10] assessed the predictive capability of the asymptotics by using Reynolds-averaged Navier-Stokes (RANS) mean flow solutions to calculate the adjoint Green's function and the low-frequency asymptotically dominant propagator term in the GAA equations. Their main numerical result (figure 5.3a) confirms that an accurate prediction of the far-field sound at low Strouhal number can be made using this asymptotic approach. The predictions generally break down (i.e., rapidly decrease), however, above the peak Strouhal number (at $S t=0.3$ or so), but that is not altogether unexpected owing to low-frequency applicability of the theory.

In this paper our aim is to further investigate the applicability of the non-parallel flow asymptotic approach by considering the effect of heating and supersonic flow using a large-eddy simulations (LES) database of two axi-symmetric round jets at a fixed jet Mach number of $M_{j}=1.5$. These solutions were reported in [11] (see also [12]) and identified by the designations B118 and B122 for the unheated and heated configurations, respectively. The operating conditions are summarized in Table 1. As opposed to our previous studies ([10] \& [14]) however, the novel aspect of this paper is that the parameters of the turbulence model for the ' 1212 ' component of the Reynolds stress auto-covariance tensor that enters the acoustic spectrum formula for the peak sound are optimized using near field turbulence data post-processed from the LES data of [12]. In this manner the model for jet noise requires only very minimal aposteriori empirical tuning. The predictions, in general, remain with $(1-2) \mathrm{dB}$ of the acoustic data up to a Strouhal number of about 0.6 for both jets. Since the turbulence model that we use (prior to optimization) for the Reynolds stress auto-covariance tensor is the only unknown here, this approach also highlights what aspects of that model can be improved upon in order to obtain better agreement with the acoustic data.

\section{Summary of asymptotic approach}

Suppose that all lengths have been normalized by the nozzle radius, $D_{j} / 2$, and all velocities by the mean jet exit velocity, $U_{j}$. Let the pressure $p$, density $\rho$, enthalpy $h$, and speed of sound $c$ satisfy the ideal gas law equation of state $p=\rho c^{2} / \gamma$ and $h=c^{2} /(\gamma-1)$, where $\gamma$ denotes the ratio of specific heats. Afsar et al. [14] applied the GSA asymptotic theory within Goldstein's generalized acoustic analogy [1] in a flow with $O(1)$ temperature fluctuations. They showed that 
the low-frequency acoustic spectrum at the observation point, $\boldsymbol{x}=\left(x_{1}, \boldsymbol{x}_{T}\right)=\left(x_{1}, x_{2}, x_{3}\right)$, due to momentum and energy transfer (via temperature fluctuations) symbolized by the generalized stress tensor $e_{\lambda j}(\boldsymbol{y}, \tau)=\left[\rho v_{\lambda}^{\prime} v_{j}^{\prime}-\overline{\rho v_{\lambda}^{\prime} v_{j}^{\prime}}\right](\boldsymbol{y}, \tau)$ in the acoustic spectrum formula, where turbulence is contained within a volume $V(\boldsymbol{y})$, is given by

$$
I(\boldsymbol{x}, \boldsymbol{y} ; \omega) \approx\left(\frac{\epsilon}{2 c_{\infty}^{2}|\boldsymbol{x}|}\right)^{2}\left[4\left|G_{12}\right|^{2}+(3-\gamma) n_{2} \operatorname{Re}\left\{\Gamma_{41} G_{11}^{*}\right\}+n_{3}\left|\Gamma_{41}\right|^{2}\right] \Phi_{1212}^{*}
$$

that is related to the Fourier transform of the far-field pressure auto-covariance, $\overline{p^{\prime}(\boldsymbol{x}, t) p^{\prime}(\boldsymbol{x}, t+\tau)}$ by

$$
I(\boldsymbol{x} ; \omega)=\int_{V_{\infty}(\boldsymbol{y})} I(\boldsymbol{x}, \boldsymbol{y} ; \omega) d \boldsymbol{y}
$$

where $|\boldsymbol{x}| \rightarrow \infty$ and $p^{\prime}(\boldsymbol{y}, \tau) \equiv p(\boldsymbol{y}, \tau)-\bar{p}(\boldsymbol{y})$. As usual, over-bars are being used to denote time averages defined by:

$$
\bar{\bullet}(\boldsymbol{x}) \equiv \lim _{T \rightarrow \infty} \frac{1}{T} \int_{0}^{T} \bullet(\boldsymbol{x}, t) d t
$$

where $\bullet$ in $(3)$ is a place holder for any fluid mechanical variable.

In the acoustic spectrum integral (2), cylindrical polar coordinates $\boldsymbol{y}=\left(y_{1}, r, \psi\right)$, commensurate with an axisymmetric round jet, are defined with respect to an origin at the nozzle exit plane. We allow normalized co-variance components, $R_{4111}=n_{2} R_{1212}$ and $R_{4141}=n_{3} R_{1212}$ where $\left(n_{2}, n_{3}\right)$ are $O(1)$ constants and suffix ' 4 ' indicates static enthalpy fluctuation via $v_{4}^{\prime}:=(\gamma-1)\left(h^{\prime}+v^{\prime 2} / 2\right) \equiv\left(c^{2}\right)^{\prime}+(\gamma-1) v^{\prime 2} / 2$ where $h^{\prime}$ is the fluctuating static enthalpy and $\left(c^{2}\right)^{\prime}$ is the fluctuations in the sound speed squared such that $v_{4}^{\prime} /(\gamma-1)$ denotes the moving frame stagnation enthalpy fluctuation [1]. Equation (1) will continue to hold for isothermal jets when mean flow quantities and turbulence parameters are appropriately defined (i.e. temperature associated correlation functions are zero: $n_{2}=n_{3}=0$ ).

\section{A. The propagator solution}

The Fourier transformed adjoint Green's functions $\left(\bar{G}_{1}, \bar{G}_{4}\right)$ represent the Green's function for the streamwise linearized momentum and energy equations of the generalized acoustic analogy ([1] \& [14]); they enter (1) through the propagator components:

$$
G_{12}=\tilde{G}_{12}(Y, U)=\frac{\partial \tilde{G}_{1}}{\partial r}-(\gamma-1) \tilde{G}_{4} \frac{\partial U}{\partial r}
$$

and, similarly,

$$
G_{11}=\bar{G}_{11}(Y, U)=\epsilon\left(\frac{\partial \bar{G}_{1}}{\partial Y}-(\gamma-1) \bar{G}_{4} \frac{\partial U}{\partial Y}\right) \quad \& \quad \Gamma_{41}=\bar{\Gamma}_{41}(Y, U)=\epsilon \frac{\partial \bar{G}_{1}}{\partial Y}
$$

that are determined for the Favre-averaged mean flow $\tilde{v}=\overline{\rho v} / \bar{\rho}=\left(U, V_{r}\right)(\boldsymbol{y})$ that depends on $y_{1}$ through the slow streamwise coordinate $Y=\epsilon y_{1}=O(1)$ for a jet of an asymptotically small spread rate, $\epsilon \ll O(1)$. The mean flow then divides into an inner region given by slowly varying mean flow expansion formulae, equations (13) -(17) in [15] (or, equations 3.1-3.4 in GSA), where $r=\left|y_{T}\right|=\sqrt{y_{2}^{2}+y_{3}^{2}}=O(1)$, and an outer region at radial distances, $R=\epsilon r=O(1)$. The propagator component $\tilde{G}_{12}(\boldsymbol{y} ; \Omega)$ is then defined at the particular scaled temporal frequency, $\Omega=\omega / \epsilon=O(1)$, shown by GSA to be where mean flow non-parallelism changes the lowest-order structure of the adjoint Green's function solution $\left(\bar{G}_{1}, \bar{G}_{4}\right)$ everywhere in the flow (and not just in the critical layer at supersonic speeds as in G\&L's solution) and at $O(1)$ Mach numbers. This distinguished limit follows supposing that the space-time adjoint vector Green's function, $g_{v 4}^{a}(\boldsymbol{y}, \tau \mid \boldsymbol{x}, t)$, depends on time, $\tau$, through the $O(1)$ slowly breathing time $\tilde{T}=\epsilon \tau$ where suffix $v=1,2 . .5$ corresponding to the 5 linearized Euler equations of the generalized acoustic analogy in [1]. In other words, as pointed out in our earlier paper [9], the Strouhal number, $S t$, is of the order of the jet spread rate, $\epsilon$, in the solution of Fourier transform of $g_{v 4}^{a}(\boldsymbol{y}, \tau \mid \boldsymbol{x}, t)($ Eqs. 7-9 in [10]).

The asymptotic structure of the adjoint Green's function $\left(\bar{G}_{1}, \bar{G}_{4}\right)$ is then identical to the mean flow in that it also divides into an inner solution in the region where the radial distance $r=O(1)$ and into an outer solution in the region where $R=\epsilon r=O(1)$. The richest inner equations are found by the non-trivial dominant balance of $g_{v 4}^{a}(\boldsymbol{y}, \tau \mid \boldsymbol{x}, t)$ given by equations 5.5 and 5.6 in GSA. The bar on $\left(\bar{G}_{1}, \bar{G}_{4}\right)$ indicates that the Green's function is a re-scaled Fourier transform 
of $g_{v 4}^{a}(\boldsymbol{y}, \tau \mid \boldsymbol{x}, t)$ for $v=(1,4)$ (see below 6 ; and corresponds to the zeroth order azimuthal mode expansion since higher-order modes produce an asymptotically small (i.e., $O(\epsilon)$ ) correction to the lowest order and therefore can be neglected at $O\left(\Omega^{0}\right)$.

[9] showed how tremendous simplification can be achieved by taking $(Y, U)$ rather than $(Y, r)$ as the independent variables of choice. The implicit function theorem shows that $\boldsymbol{y}=(Y, r)$ can be implicitly related to the field space $\boldsymbol{y}=(Y, U(Y, r))$ and that the Green's function variable $\tilde{G}_{i}(\boldsymbol{y} \mid \boldsymbol{x} ; \Omega)=\left(\tilde{G}_{1}, \tilde{G}_{4}\right)(\boldsymbol{y} \mid \boldsymbol{x} ; \Omega)$ then depends on $(\boldsymbol{y} ; \Omega)$ through field space $(Y, U(Y, r) ; \Omega) \equiv(Y, r ; \Omega)$. The one-to-one transformation of independent variables, $(Y, r) \rightarrow(Y, U)$, can be used together with the chain rule to combine the appropriate inner equations (19-21 in [10]) to the second-order hyperbolic partial differential equation

$$
\widetilde{c^{2}} \frac{\partial}{\partial U}\left(\frac{1}{\bar{c}^{2}} \bar{D}_{0} \bar{v}\right)+\bar{X}_{1} \frac{\partial^{2} \bar{v}}{\partial U^{2}}=0
$$

in which $Y=$ const., $d U / d Y=\tilde{X}_{1} / U$ are characteristic curves ([16], pp. 121-122). This equation requires that $\widetilde{c^{2}}(Y, r)=f(U)$ and satisfies Crocco's relation, and for the scaled composite Green's function variable $\bar{v}=\widetilde{c}^{2} \bar{G}_{4}+\bar{G}_{5}$ (Eq. 18 in [10]) where $\bar{G}_{5}$ corresponds to the Fourier transform of the scaled Green's function for the linearized continuity equation (Eq. 2.22a in [1]). But the Crocco-Busemann relation (see equation 2.4c in [17]), which applies when the jet flow is heated, shows that the mean speed of sound is still a function of $U(Y, r)$. Therefore, Eq.(6) will continue to hold in such a case. The advantage of solving this equation to determine the low-frequency structure of the adjoint linearized Euler equations (equations $4.8-4.10$ of [2]) is clear. The hyperbolic structure of equation (6) shows that it is unnecessary to impose a downstream boundary condition. Figure 1 in [9] indicates how information propagates to both the left and the right from the $U=0$ boundary and that no boundary conditions are required on the $Y=0$ and $Y \rightarrow \infty$ boundaries (i.e., no inflow boundary condition is required here). Hence the solution for the composite variable $v(Y, U)$ is now uniquely determined by the outer boundary conditions (i.e., by matching to the inner limit of the outer solution using Van Dyke's rule [18])

$$
\begin{gathered}
\bar{v}(Y, 0) \rightarrow-i \Omega c_{\infty}^{2} e^{-i \Omega Y \cos \theta / c_{\infty}} \\
\frac{\partial \bar{v}}{\partial U}(Y, 0) \rightarrow-i \Omega c_{\infty} \cos \theta e^{-i \Omega Y \cos \theta / c_{\infty}}
\end{gathered}
$$

on the non-characteristic curve $U=0$, with $Y \geq 0$ (where, as indicated above, $U \rightarrow 0$ corresponds to outer limit, $r \rightarrow \infty)$. The coefficient $\bar{X}_{1}$ is the streamwise component of the mean flow advection vector (equation 5.15 in [9]) and $\bar{D}_{0}=i \Omega+U \partial / \partial Y$.

The inner solution $v(Y, U)$ is then induced by incoming waves by the outer wave equation. For the $O\left(\Omega^{0}\right)$ solution, any influence of the nozzle (i.e., via the scattered wave contribution to the outer solution) can be neglected because the inner solution, which generates the scattered waves, will not behave logarithmically as $r \rightarrow \infty$ when matched to the outer solution. The logarithmic behavior of the axi-symmetric mode of the scattered solution (5.2 in [9]) follows from the small argument expansion for the solution to the two-dimensional Helmholtz equation ([19], p. 891) in the outer region. Using Van Dyke's rule, this expansion shows that $v(0, Y)$ will not match onto the transcendental $\ln R$ behavior which the solution to the Helmholtz equation induces when the inner limit is taken; i.e. as $R \rightarrow 0$ in the outer region at $O\left(\Omega^{0}\right)$ frequencies.

\section{B. Turbulence modeling}

The turbulence enters the acoustic spectrum formula (1) through the spectral tensor component $\Phi_{1212}(\boldsymbol{y} ; \omega)$ defined by the space-time Fourier transform

$$
\Phi_{1212}(\boldsymbol{y} ; \omega)=\frac{1}{2 \pi} \int_{V(\boldsymbol{\eta})} \int_{-\infty}^{\infty} R_{1212}(\boldsymbol{y}, \boldsymbol{\eta} ; \tau) e^{i(\boldsymbol{k} \cdot \boldsymbol{\eta}-\omega \tau)} d \boldsymbol{\eta} d \tau,
$$

where the $R_{1212}(\boldsymbol{y}, \boldsymbol{\eta} ; \tau)$ component of the Reynolds stress auto-covariance tensor is given by the time-average

$$
R_{1212}(\boldsymbol{y}, \boldsymbol{\eta} ; \tau)=\lim _{T \rightarrow \infty} \frac{1}{2 T} \int_{-T}^{T}\left[\rho v_{1}^{\prime} v_{2}^{\prime}-\overline{\rho v_{1}^{\prime} v_{2}^{\prime}}\right](\boldsymbol{y}, \tau)\left[\rho v_{1}^{\prime} v_{2}^{\prime}-\overline{\rho v_{1}^{\prime} v_{2}^{\prime}}\right]\left(\boldsymbol{y}+\boldsymbol{\eta}, \tau+\tau_{0}\right) d \tau_{0}
$$

\footnotetext{
*The re-scaling of Fourier transform of $g_{y 4}^{a}(\boldsymbol{y}, \tau \mid \boldsymbol{x}, t)$ via Eq. 18 in [10] for $\left.\left(\overline{\boldsymbol{G}}_{1}, \bar{G}_{4}\right), \bar{G}_{5}\right)$, which enter either the composite solution $\bar{v}=\widetilde{c^{2}} \bar{G}_{4}+\bar{G}_{5}$ determined by 6 or propagators 4 \& 5 is only necessary inasmuch as it simplifies matching conditions $7 \& 8$
} 
Construction of $R_{1212}(\boldsymbol{y}, \boldsymbol{\eta} ; \tau)$ using an exponential model with algebraic tails and the subsequent calculation of $\Phi_{1212}(\boldsymbol{y} ; \omega)$ is worked out in [10], and we use their final result

$$
\begin{aligned}
& \Phi_{1212}(\boldsymbol{y} ; \omega)=2 \pi A_{1212}(\boldsymbol{y})\left(\frac{l_{0} l_{1} l_{\perp}^{2}}{\chi^{2} U_{c}}\right) \times \\
& {\left[\left(1-a_{1}-a_{2}\right)+\left(a_{1} \tilde{\omega}^{2}-\bar{k}_{1}\left(\tilde{\omega}\left(a_{1}-a_{2}\right)\left(l_{1} / l_{0}\right)-a_{1} \bar{k}_{1}\right)\right)(4 / \chi)\right]}
\end{aligned}
$$

where $\chi\left(\tilde{\omega}, \bar{k}_{1}\right)=\bar{k}_{1}^{2}+\tilde{\omega}^{2}+1=\left(k_{1}-\tilde{\omega}\left(l_{1} / l_{0}\right)\right)^{2}+\tilde{\omega}^{2}+1$ and $\tilde{\omega}=\left(\omega l_{0} / U_{c}\right)$ is the normalized temporal frequency where $U_{c}$ is the convection velocity of the turbulence (fixed at $U_{C}=0.68$ following experiments by Harper-Bourne). The length scales in Eq. 11) are taken to be proportional to the local turbulent kinetic energy $k(y)$ and the rate of energy dissipation $\tilde{\boldsymbol{\epsilon}}(\boldsymbol{y})$ as $\boldsymbol{l}_{i}(\boldsymbol{y})=c_{i}\left(k^{3 / 2} / \tilde{\boldsymbol{\epsilon}}\right)(\boldsymbol{y})$ for $i=0,1,2,3$. We scale the amplitude of $R_{1212}(\boldsymbol{y}, \boldsymbol{\eta} ; \tau)$ as follows: $R_{1212}(\boldsymbol{y}, \mathbf{0} ; 0)=a_{1212} \bar{\rho}^{2}(\boldsymbol{y}) k^{2}(\boldsymbol{y})$ where $\bar{\rho}(\boldsymbol{y}) k(\boldsymbol{y})$ is the density-weighted RANS turbulent kinetic energy (TKE). $a_{1212}$ is usually approximated by its (maximum) value on the shear layer location $r=0.5$ at the end of the potential core of the jet ([5]). Since [14] show that $R_{1212}(\boldsymbol{y}, \boldsymbol{\eta} ; \tau)$ depends on the 5 parameters $l_{1} / l_{0}=c_{1} / c_{0}, c_{\perp}, a_{1}, a_{2}$ and $a_{1212}$ that we obtain (together with mean flow) from the LES data rather than scaling a RANS mean flow as done by [5]. For example, we show in section §. (III) that $a_{1212}$ remains largely constant in the jet. The parameters in Eq. 11, namely, length scale ratio $\left(c_{1} / c_{0}\right)$ and anti-correlation parameters $\left(a_{1}, a_{2}\right)$ are determined by using an adaptive algorithm (MP-AIDEA) to determine their optimum values against post-processed data from the LES calculations reported in [12].

Multi Population Adaptive Inflationary Differential Evolution Algorithm (MP-AIDEA, [20] \& [21]) is a populationbased evolutionary algorithm for solving single-objective global optimisation problems over continuous spaces. It combines adaptive Differential Evolution (DE) with the restarting procedure of Monotonic Basin Hopping (MBH) ([22] \& [23]). Multiple populations are initialised in the search space and exchange information during the optimisation process. Each population starts the search for the global minimum by running a DE algorithm. The user is not required to set the parameters of the DE, a process that could be extremely time consuming, since the best settings of the parameters are problem-dependent ([24] \& [25]). Instead, MP-AIDEA is able to automatically adapt these parameters during the optimisation process. At the end of the DE a local search is run from the best individual of each population. Using the restarting mechanism of $\mathrm{MBH}$ in combination with the DE, the populations are able move, in a funnel structure, from one local minima to another, until the global minimum of the problem is located. MP-AIDEA implements a novel approach to avoid multiple detection of the same local minima, by restarting the population in the entire work space when it falls within the basin of attraction of an already detected minimum. The only element of empiricism in the noise predictions, however, is in the estimation of $c_{\perp}$ that we did not analyze using the optimization algorithm. Its value, although varied by hand, turned out to be an order of magnitude smaller than $c_{1}$ (the streamwise length scale parameter), which is consistent with an axi-symmetric turbulence approximation used in this paper and analyzed more fully in [14].

\section{Optimized turbulence correlations and SPL predictions}

\section{A. Mean flow development}

The development of the mean flow components $\left(U, V_{r}\right)$ in Fig. (1) shows how heating at fixed $M_{j}$ reduces the potential core length (Figs. 1](a) \& 1(d) which is indicative of faster jet spreading. But we also see an increase in the magnitude of $X_{1}\left(y_{1}, r\right)=\bar{X}_{1}(Y, U)=V_{r} \partial U / \partial r$ along the shear layer of the jet. By measuring the slope of the upper most level curve of the streamwise mean flow in Fig. (11(a) we estimate the jet spread rates to be $\epsilon_{B 118}=0.12$ and $\epsilon_{B 122}=0.15$.

Figure 2 shows the contours of $\left|G_{12}\right|$ at the peak Strouhal number of $S t=0.2$ and downstream observation angle of $\theta=30^{\circ}$ for the B118 (Figs 2(a) \& 2(b) and B122 2(c) \& 2(d) jets. The convergence of the numerical algorithm applied to inner equation (6) has been analyzed in several of our previous papers (see [10] \& [14]), and it was found to be within 5\% at almost all regions of the jet, with only slight differences in $\partial \bar{v} / \partial U$ coming near the inner boundary as $U \gtrsim 0.7$. The verification of the Crocco and Crocco-Busemann relations for B118 and B122 jets has also been performed in our paper [14] and found to be within $5 \%$ at worst.

The peak in the momentum flux propagator, $\left|G_{12}\right|$, extends near the nozzle lip line peaking downstream at $y_{1} \sim 5-6$ compared to the subsonic jet mean flow in [10]. The reason for this is that at supersonic speeds, non-parallelism at suppresses $\left|G_{12}\right|$ from becoming singular in the critical layer at $\Omega=O(1)$ frequencies. Note that extracting the centerline streamwise velocity contour $U\left(y_{1}, 0\right)$ in Fig 1 (a) shows that the potential core lengths correspond to a streamwise length scale $y_{1}$ of approximately $L_{B 118} \sim 4$ and $L_{B 118} \sim 2.5$ Heating at fixed $M_{j}$ therefore concentrates the peak propagator 

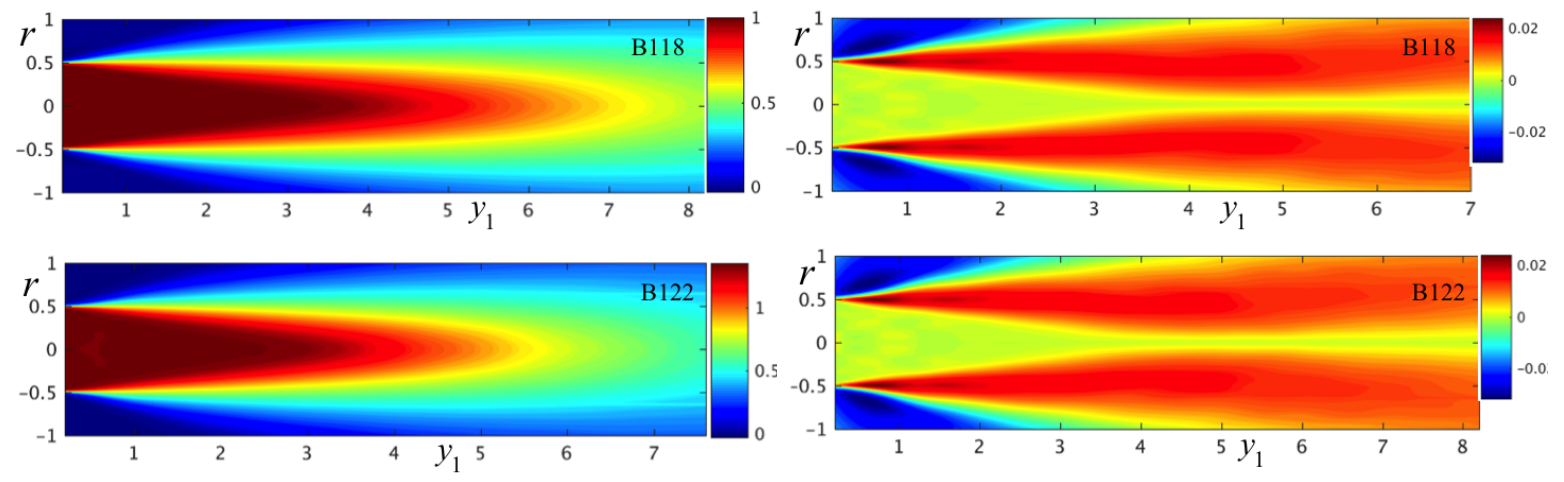

(a) Streamwise mean flow, $U\left(y_{1}, r\right)$

(b) Radial mean flow, $V_{r}\left(y_{1}, r\right)$
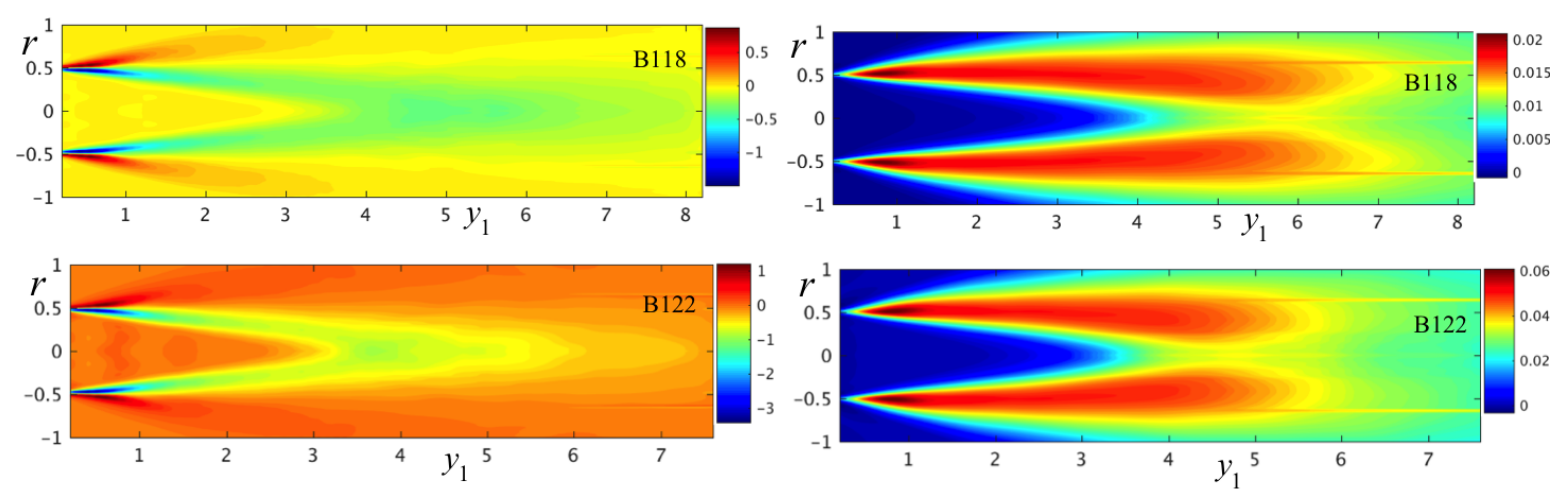

(c) Stremwise mean flow advection, $\bar{X}_{1}\left(y_{1}, r\right)$

(d) Turbulent kinetic energy, $\bar{\rho} k\left(y_{1}, r\right)$

Fig. 1 Mean flow development for B118 and B122 jets.

$\left|G_{12}\right|$ to lie within one potential core length of the jet (cf. Figs:2(a) \& 2(c)]. In Figs. 2(b) \& 2(d) shows contour plots of the radius-weighted acoustic spectrum $r I_{L O W}$ using one set of optimized scales for $\left(l_{r}, a_{1}, a_{2}\right)$ that shall be discussed soon. The indication here is that the peak noise source lies just beyond the end of the potential cores of both jets (cf. streamwise potential core lengths above) such that the maximum contour level of $r I(\boldsymbol{x}, \boldsymbol{y} ; \omega)$ is greater with heating at fixed $M_{j}$. While, at its greatest, this stands at an almost 2 order of magnitude increase in $r I(x, y ; \omega)$ of $10^{-8}$ (Fig 2(b)) compared to $10^{-10}$ for B118 (Fig 2(d)), this intense region in B122 is much thinner in $r$ and more localized within the potential core in $y_{1}$ in Fig. (2(d)), hence it is likely that the surrounding (more blue) contours where $r I(\boldsymbol{x}, \boldsymbol{y} ; \omega)$ $\sim(0.2-0.3) \times 10^{-8}$ contribute more significantly to 2). This is then consistent with the almost $5-6 \mathrm{~dB}$ increase in sound for these jets that the noise measurements of [26] indicate.

Figure 3 shows that LES extracted ratio $R_{1212}(\boldsymbol{y}, \mathbf{0} ; 0) / \bar{\rho}^{2}(\boldsymbol{y}) k^{2}(\boldsymbol{y})=a_{1212}$ determined at the shear layer $r=0.5$ remains more-or-less constant in the jet. Hence we fix $a_{1212}=0.45$ for B118 and $a_{1212}=0.5$ for B122. Figure 4 shows the outcome of optimizing $\left(l_{r}, a_{1}, a_{2}\right)$ in:

$$
\begin{aligned}
\frac{R_{1212}\left(\boldsymbol{y}, \eta_{1}, 0, \tau\right)}{R_{1212}(\boldsymbol{y}, \mathbf{0}, 0)} & = \\
& {\left[1+a_{1} \frac{\tilde{\tau}}{X} l_{r}^{2}\left(\tilde{\eta}_{1}-\tilde{\tau}\right)-a_{2} \frac{\tilde{\eta}_{1}}{X}\left[\tilde{\eta}_{1}+l_{r}^{2}\left(\tilde{\eta}_{1}-\tilde{\tau}\right)\right]+\ldots\right] e^{-X} }
\end{aligned}
$$

where $X=X\left(\tilde{\eta}_{1}, \tilde{\eta}_{T}, \bar{\tau}\right)=\sqrt{\tilde{\eta}_{1}^{2}+|\tilde{\xi}|^{2}}, \tilde{\eta}_{1}=\eta_{1} / l_{1}$ and $\bar{\tau}=U_{c} \tau / l_{1}$ allows the space-time dependence of 12 to enter 


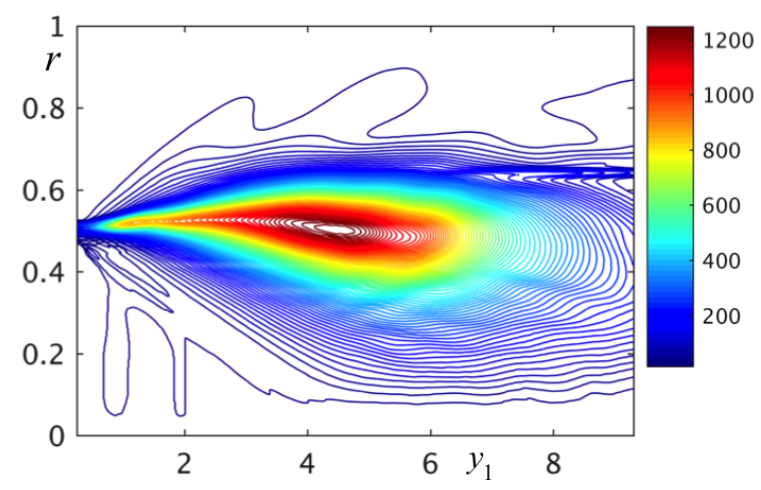

(a) B118: $G_{12}\left(y_{1}, r\right)$

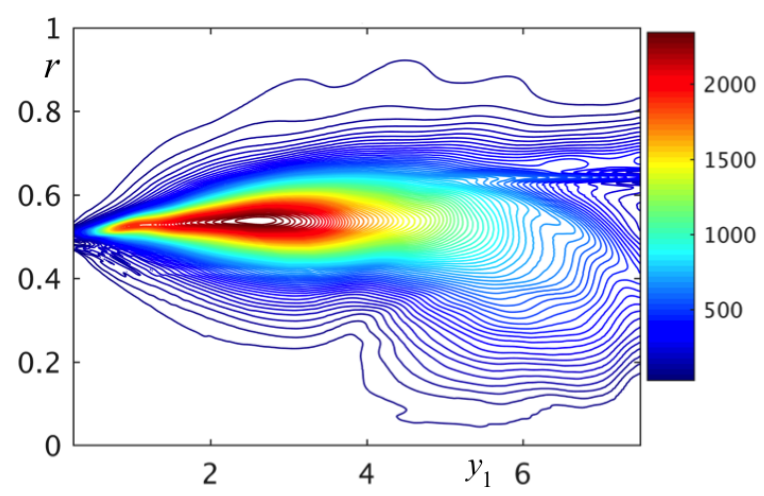

(c) B122: $G_{12}\left(y_{1}, r\right)$

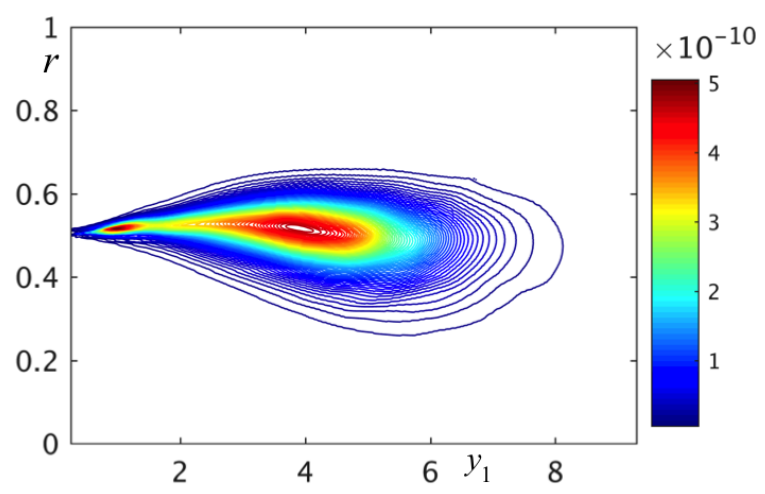

(b) B118: $r I(\boldsymbol{x}, \boldsymbol{y} ; \omega)$

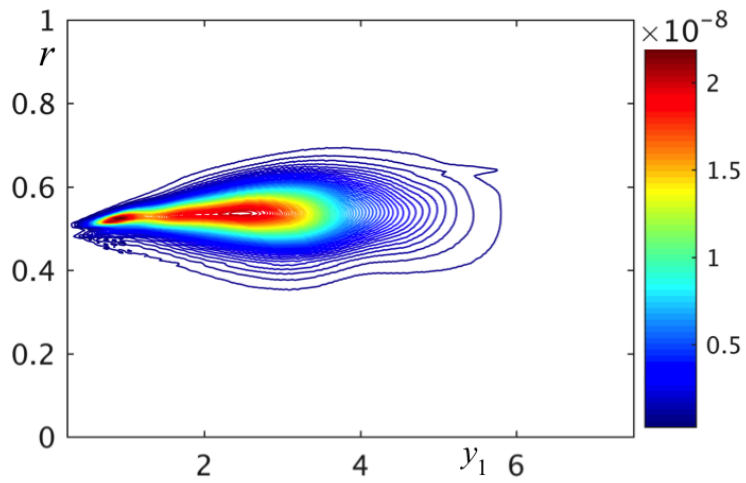

(d) B122: $r I(x, y ; \omega)$

Fig. 2 Spatial structure of momentum flux propagator $G_{12}\left(y_{1}, r\right),(4)$, and radius-weighted acoustic spectrum $r I(\boldsymbol{x}, \boldsymbol{y} ; \omega),(\mathbf{1})$, for $\mathrm{B} 118$ and $\mathrm{B} 122$ jets.

only through the scaled non-dimensional convected variable, $\tilde{\xi}=l_{r}\left(\tilde{\eta}_{1}-\bar{\tau}\right)$ where $l_{r}=l_{1} / l_{0}=c_{1} / c_{0}$.

We applied the MP-AIDEA algorithm in two ways. First by optimizing the $R_{1212}\left(y, \eta_{1}, 0, \tau\right)$ model, $[12)$, at all $\tilde{\eta}_{1}=(0.0,0.3,0.6)$ (case a) and then at $\tilde{\eta}_{1}=0.0$ only (case b). The results in Figure 4 indicate that whilst case $\mathrm{b}$ guarantees perfect agreement at $\tilde{\eta}_{1}=0.0$ (Figs. 4(b) \& 4(d) it introduces a departure at $\tilde{\eta}_{1}=(0.3,0.6)$. On the other hand, for case a, (12) gets the right maximum height from $\tau=0$ axis, but the location of the peaks are shifted. But the acoustic predictions using these set of parameters for cases $a \& b$ do not result in significant change for B118 (Fig 5(a)] and a difference of $(1-3) \mathrm{dB}$ for B122 (Fig $5(\mathrm{~b})$. Note that just as in the jet noise calculations in Afsar et al. (2019), we show in the appendix that a heated jet at fixed $M_{j}$ displays predictions for the peak sound that are more-or-less independent of $\left(n_{2}, n_{3}\right)$ in (1). The B122 predictions in Figs. 5 \& 6 are therefore taken at $\left(n_{2}, n_{3}\right)=0$. Given the fact the case $b$ optimized parameters for $R_{1212}\left(y, \eta_{1}, 0, \tau\right)$ where in agreement with case $a$ for the $30^{0}$ prediction in Fig 5 . we concentrate on this choice of parameters in Fig. 6. Here we show the predictions at various downstream angles $\theta=\left(30^{\circ}, 35^{\circ}, 40^{\circ}\right)$ to assess the limit of applicability of the asymptotic approach. It is evident that the predictions show greater departure to the acoustic data as $\theta$ increases away from the peak noise location, which is consistent with the distinguished asymptotic scaling derived in [9] \& [14] having less validity at larger angles (i.e. that non-parallel flow effects are no longer affect the lowest order Green's function solution at these angles). 


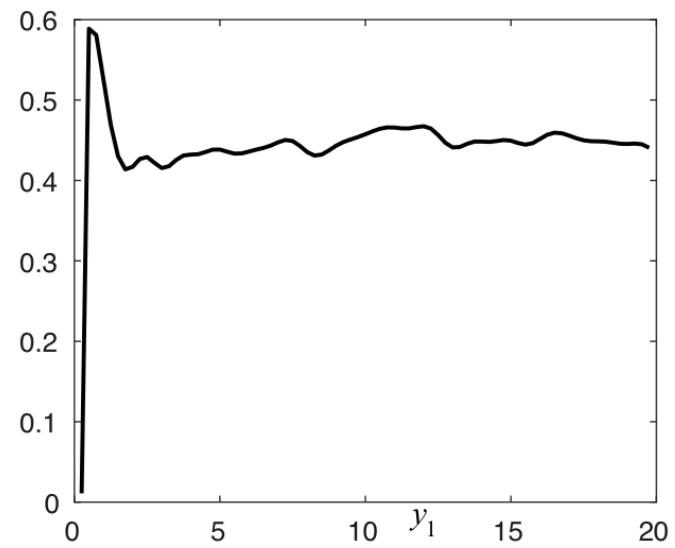

(a) B118

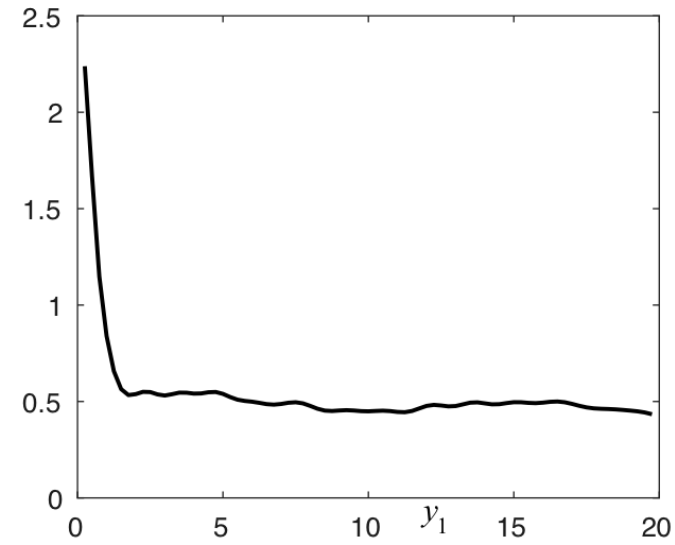

(b) B122

Fig. 3 Streamwise development of $R_{1212}(\boldsymbol{y}, \mathbf{0} ; 0) / \bar{\rho}^{2}(\boldsymbol{y}) k^{2}(\boldsymbol{y})$ at $r=0.5$ for $\mathrm{B} 118$ and $\mathrm{B} 122$ jets.

$\begin{array}{llllllll}\text { Jet } & \text { Optimization case } & l_{r} & a_{1} & a_{2} & c_{0} & c_{1} & c_{\perp} \\ \mathrm{B} 118 & \mathrm{a} & 0.56 & 0.37 & 1.0 & 0.3 & 0.176 & 0.075 \\ \mathrm{~B} 118 & \mathrm{~b} & 0.53 & 0.36 & 1.29 & 0.35 & 0.184 & 0.1 \\ \mathrm{~B} 122 & \mathrm{a} & 0.432 & 0.277 & 1.0 & 0.3 & 0.130 & 0.034 \\ \mathrm{~B} 122 & \mathrm{~b} & 0.965 & 0.0 & 0.688 & 0.3 & 0.289 & 0.017\end{array}$

Table 2 Optimized turbulence scales used in prediction via (12), (11), (1) \& (2)

\section{Conclusion}

The use of a nearly optimized turbulence model for the appropriate Reynolds stress auto-covariance tensor component $R_{1212}(\boldsymbol{y}, \boldsymbol{\eta} ; \tau)$ that enters the peak noise formula (1) results in acoustic predictions that are very accurate beyond what would normally constitute the low frequency regime. Our results show the robustness of the asymptotic approximation for the adjoint vector Green's function of the linearized Euler equations in the presence of slowly diverging mean flow worked out by Goldstein-Sescu-Afsar [9] and re-formulated in [14]. In this paper we have extended the work of [10] and [14] to assess the validity of this approach for axi-symmetric flows of fixed jet Mach number. Our results have shown that the theory not only provides a means to understand, qualitatively, the effects of non-parallelism within the acoustic analogy but also provides excellent predictive capability of the jet noise. The reduced form of the acoustic spectrum formula (equation 19 in [4]) used here is limited to low frequencies and shallow downstream observation angles from the jet axis. The results in this paper indicate that the $30^{\circ}$ spectrum can be accurately predicted in both the unheated (B118) and heated (B122) cases up to a Strouhal number of almost 0.5. Future work will involve investigating different optimization techniques to better model $R_{1212}$ auto-covariance.

\section{Acknowledgments}

This work began when the authors were visiting the Stanford Center for Turbulence Research summer program 2016. We would, therefore, like to thank Professor Parviz Moin for providing the initial impotus to start this. We warmly acknowledge the assistance provided by Profs. Joe Nichols (University of Minnesota) and Sanjiva Lele and Dr. Guillaume Bres in the initial post-processing of the LES data. We would also like to thank Dr. S. J. Leib (Ohio Aerospace Institute) for providing us with the spectral tensor routines. Computational resources from HPC2, Mississippi State University, are appreciated. MZA would like to thank Strathclyde University for financial support from the Chancellor's Fellowship. 


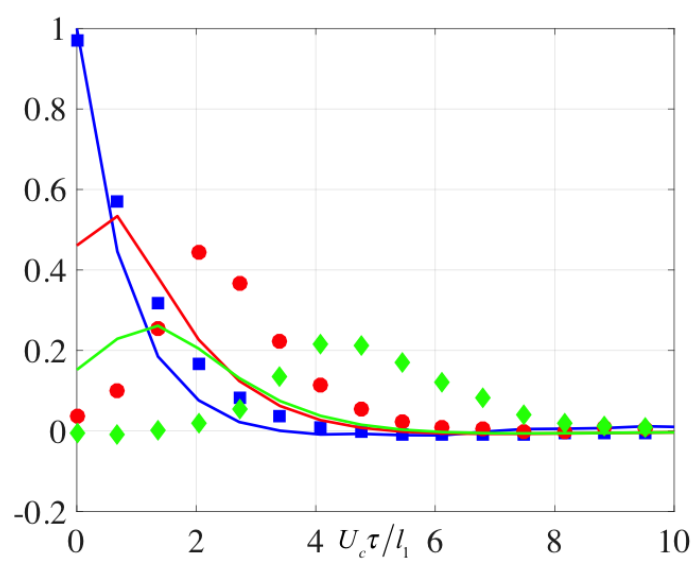

(a) B118: case a (optimizing at all $\left.\eta_{1}\right)$

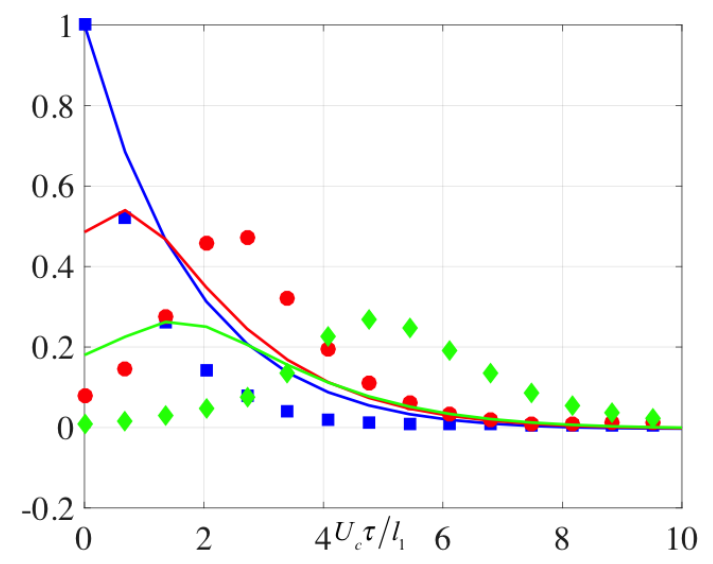

(c) B122: case a

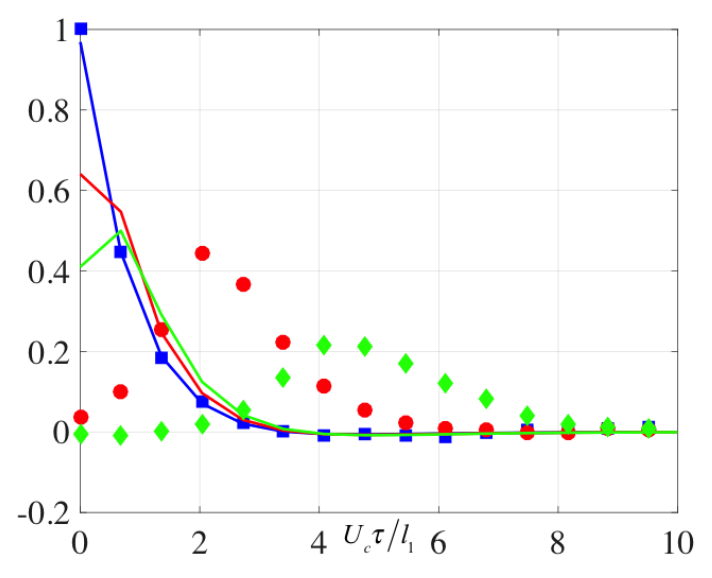

(b) B118: case b ((optimizing at $\left.\left.\eta_{1}=0\right)\right)$

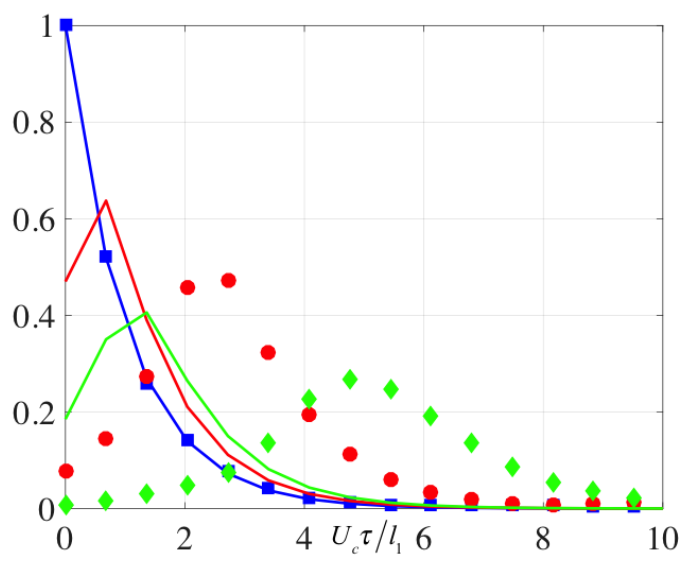

(d) B122: case b

Fig. 4 Application of MP-AIDEA algorithm to optimization of $R_{1212}\left(y, \eta_{1}, 0, \tau\right)$ using (12) against LES data [12].

\section{Appendix A: Effect of $n_{2}=n_{3} \neq 0$ in (1)}

In Fig.(7) we show the effect on the peak acoustic spectrum of taking $n_{2}=n_{3} \neq 0$ in (1). As in the fixed acoustic Mach number heated jet predictions in [14], our results show little (if any) sensitivity to $\left(n_{2}, n_{3}\right)$ parameters in the acoustic spectrum formula (1).

\section{References}

[1] Goldstein, M. E., “A generalized acoustic analogy.” J. Fluid Mech., Vol. 488, 2003, pp. 315-333.

[2] Goldstein, M. E., and Leib, S. J., “The aero-acoustics of slowly diverging supersonic jets.” J. Fluid Mech., Vol. 600, 2008, pp. 291-337.

[3] Afsar, M. Z., "Asymptotic properties of the overall sound pressure level of sub-sonic air jets using isotropy as a paradigm," $J$. Fluid Mech., Vol. 8, No. 2, 1997, pp. 13-19.

[4] Afsar, M. Z., Goldstein, M. E., and Fagan, A. M., "Enthalpy-flux/momentum-flux coupling in the acoustic spectrum of heated jets," AIAA J, Vol. 49, 2011, pp. 2252-2262.

[5] Karabasov, S., Afsar, M., Hynes, T., Dowling, A., McMullan, W., Pokora, C., Page, G., and McGuirk, J., "Jet noise: acoustic analogy informed by large eddy simulation," AIAA Journal, Vol. 48, 2010, pp. 1312-1325. 


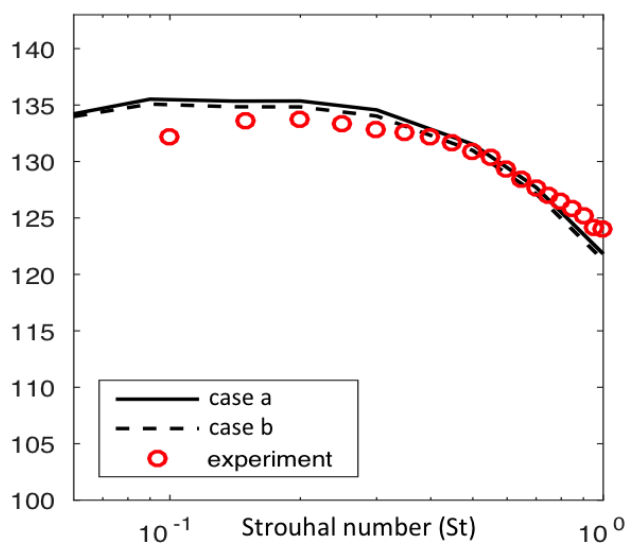

(a) B118

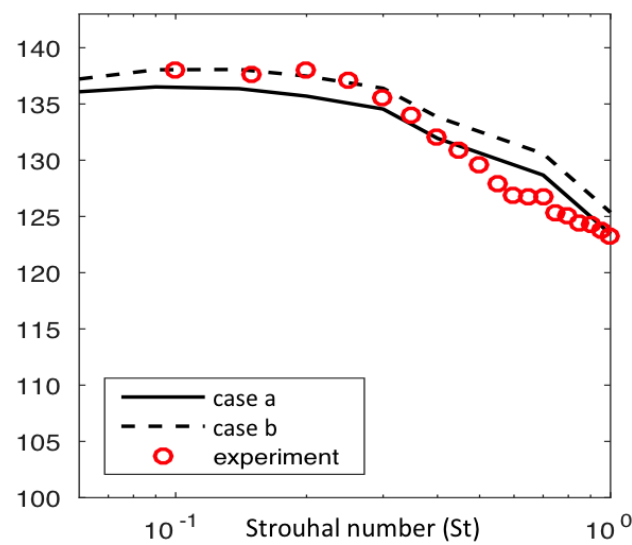

(b) B122

Fig. 5 SPL prediction against acoustic data in [26]. SPL computed via $S P L=10 \log _{10} 4 \pi\left(\rho U_{J}^{2}\right)^{2} I(x ; \omega) / P_{r e f}^{2}$ where $P_{\text {ref }}=2 \times 10^{-5}$ Pa. The spectrum $I(x ; \omega)$ is determined by integrating $(1)$ over $\boldsymbol{y}=\left(\mathbf{y}_{1}, \mathbf{r}, \phi\right)$ where turbulence scales in $\Phi_{1212}^{*}\left(y, k_{1}, k_{T}^{2} ; \omega\right),(11)$ summarized in table 2

[6] Goldstein, M. E., "The low frequency sound from multipole sources in axysymmetric shear flows with application to jet noise." J. Fluid Mech., Vol. 70, 1975, pp. 595-604.

[7] Afsar, M. Z., "Insight into the two-source structure of the jet noise spectrum using a generalized shell model of turbulence." Euro. J. Mech. B/Fluids, Vol. 31, 2012, pp. 129-139.

[8] Karabasov, S. A., Bogey, C., and Hynes., "Computation of noise of initially laminar jets using a statistical approach for the acoustic analogy: application and discussion," AIAA 2011-2929, 2011.

[9] Goldstein, M. E., Sescu, A., and Leib, S. J., "Effect of non-parallel mean flow on the Green's function for predicting the low frequency sound from turbulent air jets." J. Fluid Mech., Vol. 695, 2012, pp. 199-234.

[10] Afsar, M. Z., Sescu, A., and Leib, S. J., "Predictive capability of low frequency jet noise using an asymptotic theory for the adjoint vector Green's function in non-parallel flow," AIAA Paper, Vol. 2016-2804, 2016.

[11] Brès, G. A., Nichols, J. W., Lele, S. K., , and Ham, F., "Towards best practices for jet noise predictions with unstructured large eddy simulations," AIAA Paper 2012-2965, 2012.

[12] Brès, G., Ham, F., Nichols, J., and Lele, S., “Unstructured large eddy simulations of supersonic jets,” AIAA J, 2016.

[13] Schlinker, R. H., Simonich, J. C., Reba, R. A., Colonius, T., Gudmundsson, K., and Ladeinde, F., "Supersonic Jet Noise from Round and Chevron Nozzles: Experimental Studies," AIAA 2009-3257, 2009.

[14] Afsar, M. Z., Sescu, A., and Sassanis, V., "Validity of a non-parallel flow asymptotic theory for the adjoint Green's function within the generalized acoustic analogy: its role in explaining the 'quietening' of heated supersonic jets," Submitted to Phys. Fluids, 2019.

[15] Afsar, M. Z., Sescu, A., and Leib, S. J., "Predictive Capability of Low Frequency Jet Noise using an Asymptotic Theory for the Adjoint Vector Green's Function in Non-parallel Flow," AIAA 2016-2804, 2016.

[16] Garabedian, P. R., Partial Differential Equations, AMS Chelsea Publishing, New York, 2008.

[17] Leesshafft, L., Huerre, P., Sagaut, P., and Terracol, M., "Nonlinear global modes in hot jets.” J. Fluid Mech., Vol. 554, 2006, pp. 393-409.

[18] Van Dyke, M., Perturbation Methods in Fluid Mechanics, Parabolic Press, Stanford Univ., 1975.

[19] Morse, P. M., and Feshbach, H., Methods of Theoretical Physics, McGraw-Hill, New York, 1953. 
[20] Di Carlo, M., Vasile, M., and Minisci, E., "Multi-Population Adaptive Inflationary Differential Evolution Algorithm with Adaptive Local Restart," IEEE Congress on Evolutionary Computation CEC, Sendai, Japan, 2015.

[21] Di Carlo, M., Vasile, M., and Minisci, E., "Multi-Population Adaptive Inflationary Differential Evolution Algorithm," BIOMA (Bio-Inspired Optimisation Methods and their Applications) Workshop, Ljubljana, Slovenia, 2014.

[22] Wales, D. J., and Doye, J. P. K., "Global optimization by basin-hopping and the lowest energy structures of Lennard-Jones clusters containing up to 110 atoms," J. Phys. Chem. A, Vol. 101, 1997, pp. 5111-5116.

[23] Addis, B., Locatelli, M., and Schoen, F., "Local optima smoothing for global optimization," Optimization Methods and Software, Vol. 20, 2005, p. 4170437.

[24] Minisci, E., and Vasile, M., "Adaptive Inflationary Differential Evolution,” IEEE Congress on Evolutionary Computation CEC, Sendai, Japan, 2014.

[25] Vasile, M., Minisci, E., and Locatelli, M., "An inflationary differential evolution algorithm for space trajectory optimization," IEEE Transactions on Evolutionary Computation, Vol. 15 (2), 2011, pp. 267-281.

[26] Schlinker, R. H., Simonich, J. C., Reba, R. A., Colonius, T., Gudmundsson, K., and Ladeinde, F., "Computation of noise of initially laminar jets using a statistical approach for the acoustic analogy: application and discussion,” AIAA 2009-3257, 2009. 


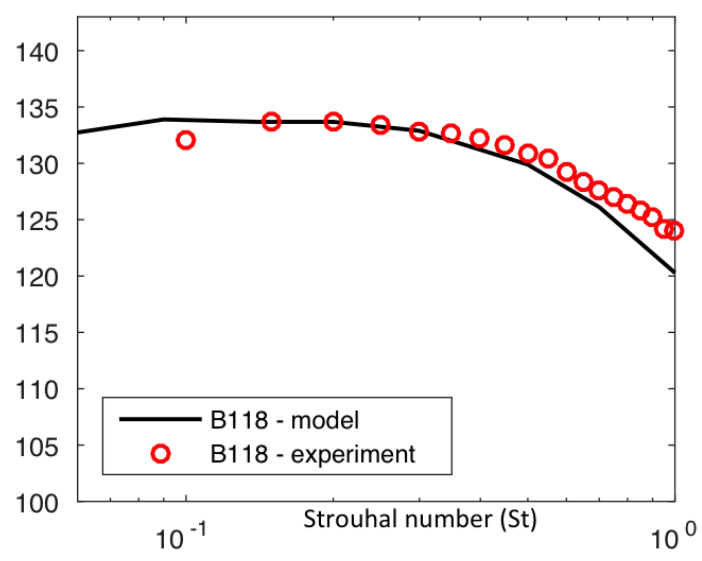

(a) B118: $\theta=30^{\circ}$

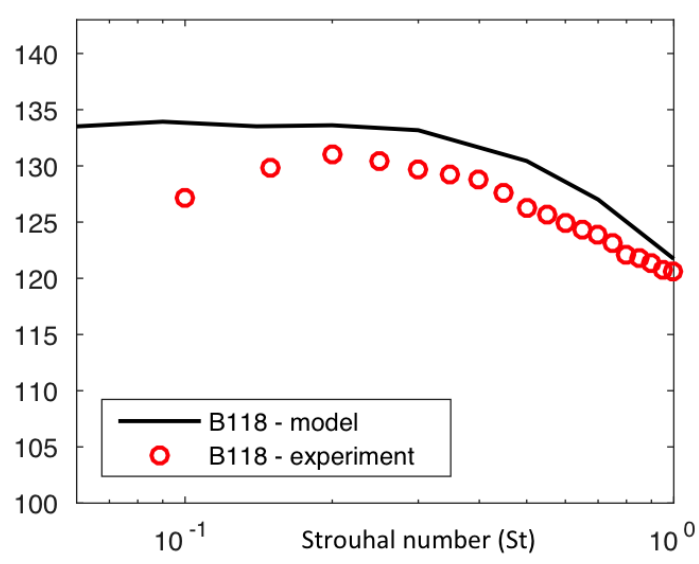

(c) B118: $\theta=40^{0}$

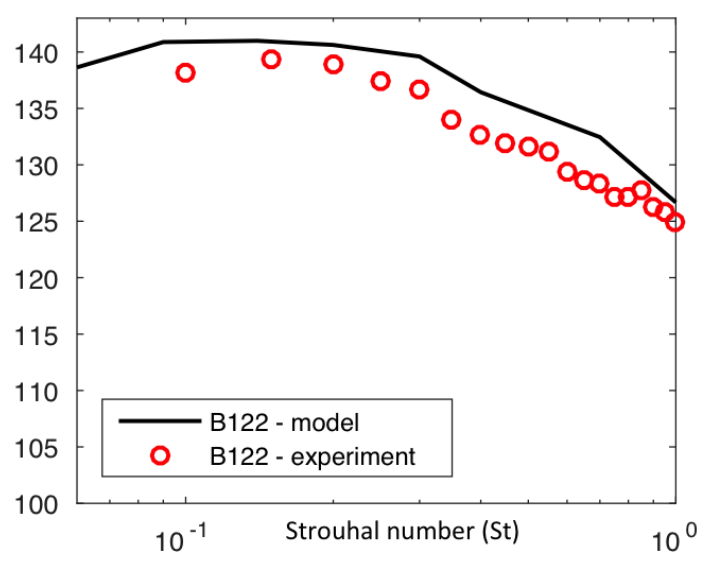

(e) $\mathrm{B} 122: \theta=35^{0}$

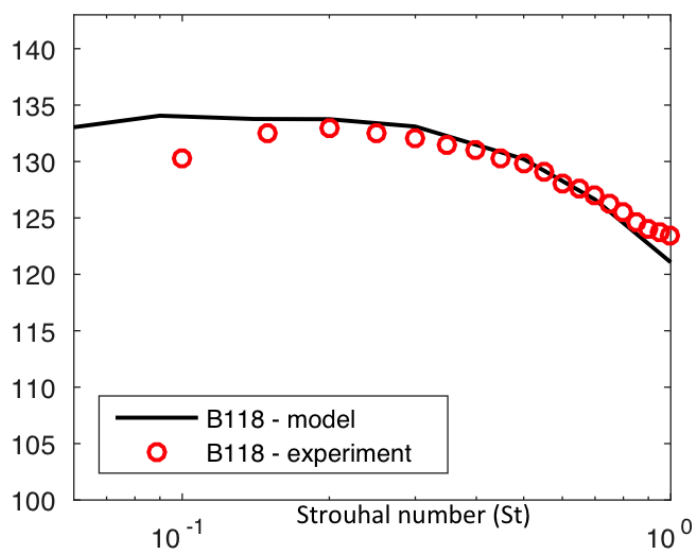

(b) $\mathrm{B} 118: \theta=35^{0}$

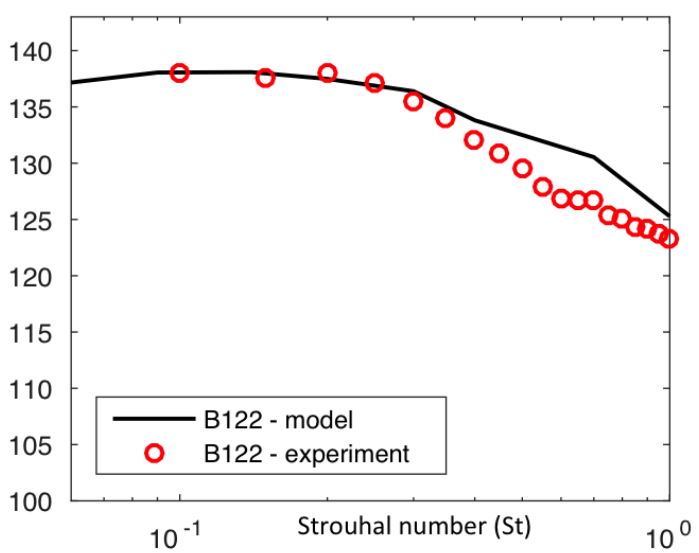

(d) B122: $\theta=30^{0}$

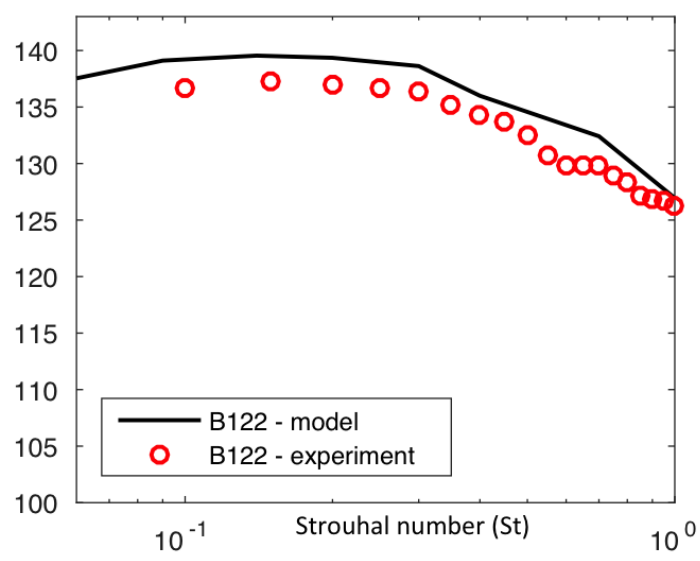

(f) $\mathrm{B} 122: \theta=40^{0}$

Fig. 6 SPL prediction against acoustic data in [26]. SPL computed as caption as Fig. (5). Turbulence scales in $\Phi_{1212}^{*}\left(\boldsymbol{y}, k_{1}, k_{T}^{2} ; \omega\right), \mathbf{1 1}$ summarized in table 2 


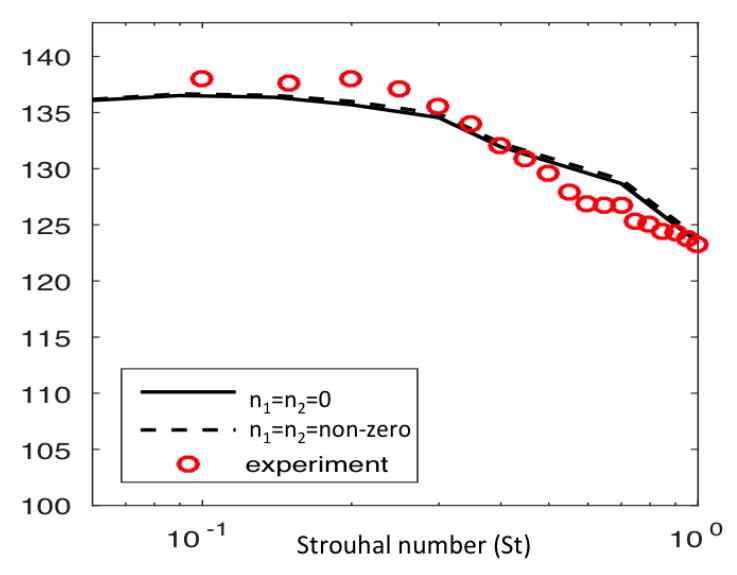

(a) Case a optimized parameters in 12

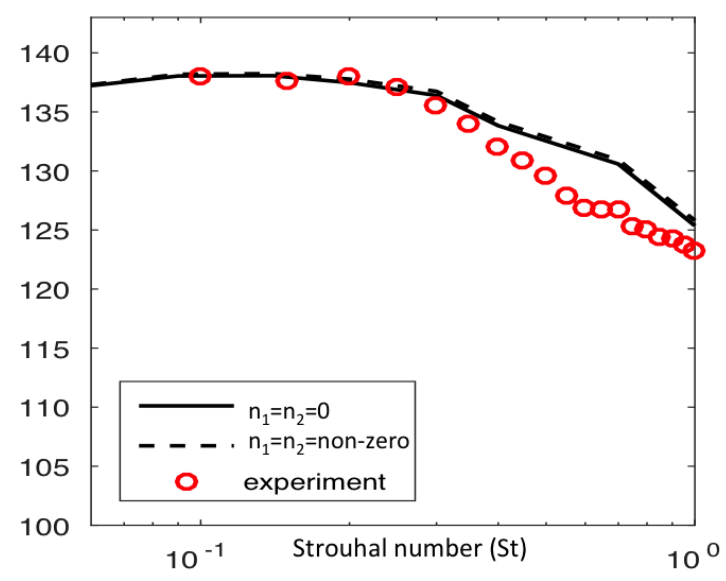

(b) Case b

Fig. 730 degree prediction for B122 jet against acoustic data in [26]. SPL computed as in Fig. (5). Turbulence scales in $\left.\Phi_{1212}^{*}\left(y, k_{1}, k_{T}^{2} ; \omega\right), 11\right)$ summarized in table 2 